\title{
Vacuum-assisted closure vs. bilateral pectoralis major muscle flaps for deep sternal wounds infection
}

\author{
Tuo Pan ${ }^{1}$, Kai Li ${ }^{1}$, Fu-Dong Fan ${ }^{1}$, Yong-Shun Gao ${ }^{2}$, Dong-Jin Wang ${ }^{1}$ \\ ${ }^{1}$ Department of Cardio-Thoracic Surgery, Nanjing Drum Tower Hospital, The Affiliated Hospital of Nanjing University Medical School, \\ Nanjing 210008, China; ${ }^{2}$ Department of Cardiovascular Surgery, The Seventh Medical Center of People's Liberation Army General Hospital, \\ Beijing 100010, China \\ Contributions: (I) Conception and design: YS Gao, DJ Wang; (II) Administrative support: K Li; (III) Provision of study materials or patients: FD Fan; \\ (IV) Collection and assembly of data: T Pan; (V) Data analysis and interpretation: T Pan; (VI) Manuscript writing: All authors; (VII) Final approval \\ of manuscript: All authors. \\ Correspondence to: Prof. Dong-Jin Wang. Chief Director of the Department of Cardio-Thoracic Surgery, Nanjing Drum Tower Hospital, Number \\ 321 Zhongshan Road, Nanjing 210008, China. Email: dongjin_wang@126.com; Prof. Yong-Shun Gao. Director of the Department of Cardiovascular \\ Surgery, The Seventh Medical Center of People's Liberation Army General Hospital, Number 5, Nanmencang, Dongcheng District, Beijing 100010, \\ China. Email: Yongshun_Gao@126.com.
}

Background: Deep sternal wound infection (DSWI) is a life-threatening complication after cardiac surgery. The aim of this study was to retrospectively evaluate the outcomes of reconstructing infected poststernotomy wounds with either vacuum-assisted closure (VAC) after previous debridement or bilateral pectoralis major muscle flaps (BPMMFs).

Methods: In total, 565 patients with postoperative DSWI were enrolled in this study from January 1, 2014, to June 1, 2018. Of these patients, 247 received BPMMFs. To address the indicated biases, a 1:1 propensity score-matched cohort was created based on age, body mass index, preoperative diabetes mellitus, chronic obstructive pulmonary disease (COPD), internal thoracic artery grafting (ITAG), type of cardiac surgery, time before treatment for DSWI and wound classification. After matching, 132 patients who had similar risk profiles were enrolled in the study population (66 in the VAC group: 66 in the BPMMF group).

Results: At 21.9 \pm 12.1 (median: 24, IQR: 14-28) months of follow-up, the survival rate was $93.9 \%$ in the BPMMF group and $74.4 \%$ in the VAC group $(\mathrm{P}<0.01)$. Compared with the VAC group, the BPMMF group had a significantly decreased length of hospital stay $(\mathrm{P}<0.01)$. At the spirometry assessment, the forced expiratory volume in the 1st second (FEV1), vital capacity (VC), and FEV1/VC ratio showed no significant differences in survival between the VAC group and BPMMF group.

Conclusions: In our study, compared with VAC therapy, BPMMFs guaranteed better early- and lateterm outcomes, as shown by less length of hospital stay, a higher rate of long-term survival and unimpaired respiratory function.

Keywords: Deep sternal wound infection (DSWI); vacuum-assisted closure therapy; bilateral pectoralis major muscle flaps (BPMMFs)

Submitted Sep 16, 2019. Accepted for publication Dec 05, 2019.

doi: $10.21037 /$ jtd.2019.12.76

View this article at: http://dx.doi.org/10.21037/jtd.2019.12.76

\section{Introduction}

Deep sternal wound infection (DSWI) is a rare event after cardiac surgery $(1,2)$. In a recent series of reports, the reported incidence of DSWI ranged from $1 \%$ to $4 \%(2-4)$.
DSWI significantly increases mortality, morbidity and hospital costs $(1,5)$.

Several studies have reported risk factors for DSWI, including obesity, 71 advanced age, chronic obstructive 
pulmonary disease (COPD), diabetes and use of internal thoracic artery conduits $(1,3,6,7)$. Other studies have explored various therapeutic options for postoperative DSWI $(4,8,9)$. These include debridement with early or delayed closure, vacuum-assisted closure (VAC), and partial or complete sternotomy with reconstruction using a flap $(4,8,9)$. Owing to these previous studies, the clinical strategies for the treatment of DSWI (both surgical procedures and postoperative care) have undergone rapid progress in recent years, resulting in improved mortality and morbidity (10). Among these strategies, the most popular methods for the treatment of DSWI is VAC therapy (11).

VAC therapy is based on a fixed negative pressure applied to the wound, resulting in drainage of the wound fluid and granulation. An increasing number of centers have reported that VAC therapy may significantly improve outcomes (4,12-14). A retrospective study indicated that aggressive sternal debridement followed by VAC therapy and secondary closure with an omental muscle flap is effective for DSWI (15). Therefore, VAC therapy is a useful and eligible method to treat serious infections. However, However, Biefer et al. reported that VAC therapy alone does not reduce the 85 length of ICU stay and the length of hospital stay compared with the corresponding stays after direct wound closure (16). Moreover, Eyileten et al. reported that VAC might be considered for patients without osteomyelitis but a stable sternum (17). Therefore, there remains a lack of consensus regarding VAC therapy.

Bilateral pectoralis major muscle flaps (BPMMFs) for surgical treatment of DSWI were used in our hospitals from 1991. Then, in 2001, we first reported that BPMMFs could significantly improve outcomes in patients with DSWI after cardiac surgery (18). After nearly three decades of effort, clinical practices have fully proven that BPMMFs are reliable and appropriate for the treatment of DSWI without additional incisions (19). However, compared with VAC therapy, which is currently prevailing in the management of postoperative DSWI, the effect of BPMMFs in patients with DSWI is still unknown. Therefore, we conducted a retrospective study to evaluate the results of reconstructing infected poststernotomy wounds with either VAC therapy or BPMMFs.

\section{Methods}

\section{Study population}

The study was conducted in Nanjing Drum Tower Hospital and the Seventh Medical Center of People's Liberation Army (PLA) General Hospital. This study was approved by ethics committee of Nanjing Drum Tower Hospital (No. 2019-244-01). After the ethical committees of Nanjing Drum Tower Hospital approved the study, we started this retrospective study on January 1, 2014. Adults (age at least 18 years) who had developed a postoperative DSWI between January 1, 2014, and June 1, 2018, and who were followed up at least 4 months after BPMMF placement (BPMMF group) or VAC therapy (VAC group) were considered eligible. Patients who died within 7 postoperative days were not included in the evaluation.

In our opinion, DSWI presents in one of three different ways. The earliest presentation occurs in patients with wound separations with or without sternal instability that develop within a few days of sternotomy (20). The second method of presentation involves a fulminant mediastinitis usually within the first few weeks after operation (20). The last type of infected sternotomy is the chronically infected wound of several weeks' or several months' duration (20). According to our local protocol, the VAC therapy + aggressive debridement was usually implemented in patients with earliest DSWI; BPMMFs was routinely used in patients with chronical DSWI. However, patients who had second presentation of DSWI were controversially treated. These patents were treated by different methods, including isolated VAC therapy, isolated BPMMF or VAC therapy + BPMMF. Between January 1, 2014, and June 1, 2018, 565 patients with postoperative DSWIs were referred to our hospitals for treatment. All sternal infections met the criteria of the Centers for Disease Control and Prevention (21). In the case of visual evidence of mediastinitis during the operation $(\mathrm{n}=122)$, the bacterium involved was isolated from the culture of mediastinal tissue or fluid $(n=360)$, and fever or instability of the sternum was associated with purulent drainage from the mediastinum $(n=83)$. There were 247 patients who received BPMMFs. These 247 patients had not received VAC therapy before undergoing BPMMF placement. The other 318 patients received VAC therapy. Furthermore, after VAC therapy, 89 patients with uncontrolled DSWIs or the recurrence of DSWI underwent a secondary BPMMF, and the remaining 229 patients underwent delayed primary closure.

To address potential biases, a 1:1 propensity scorematched cohort was created based on age, body mass index (BMI), preoperative diabetes mellitus, COPD, internal thoracic artery grafting (ITAG), type of cardiac surgery, time before treatment for DSWI and wound classification (20). 


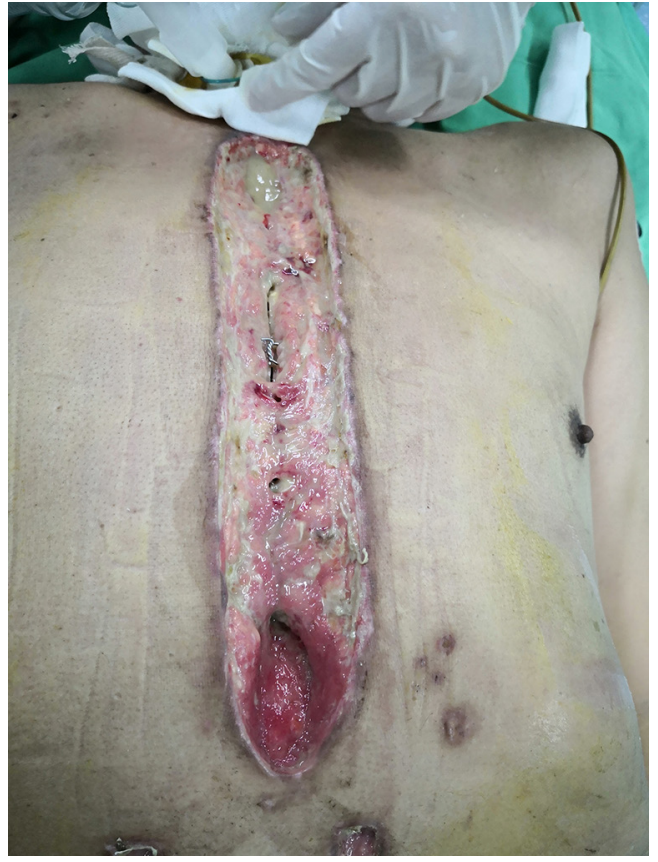

Figure 1 Bilateral pectoralis major muscle flaps: diagnosis of deep sternal wound infection.

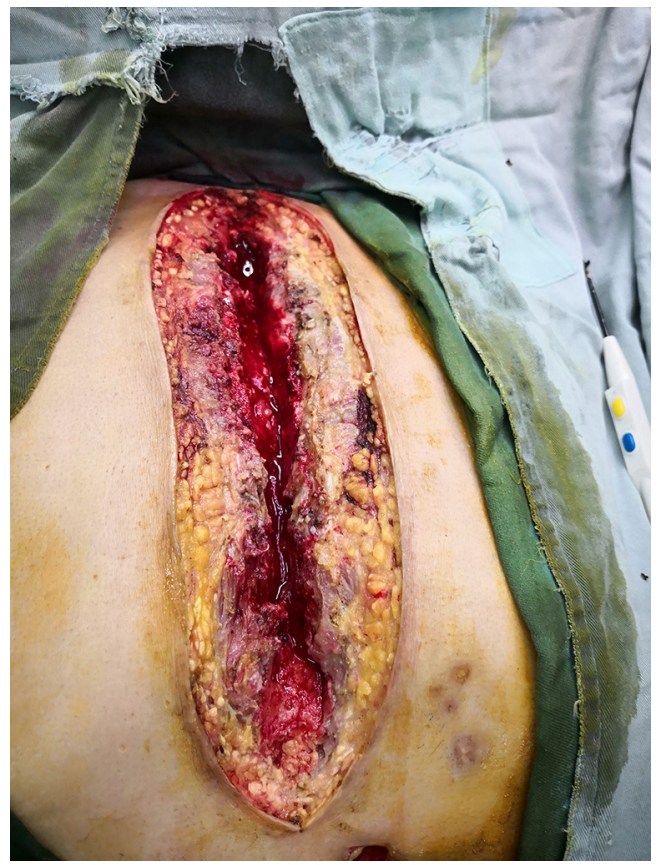

Figure 2 Bilateral pectoralis major muscle flaps: all sternal wires as well as devascularized and necrotic cutaneous, bone and cartilage tissues were resected.
After matching, 132 patients who had similar risk profiles were enrolled in the study population (66 in the VAC group: 66 in the BPMMF group).

\section{Study procedures}

After the diagnosis of DSWI, patients in the VAC group first underwent wound incision under aseptic conditions. Then, aggressive debridement was performed on these patients. After debridement, all patients received VAC therapy (KCI, West San Antonio, Texas, USA) for wound closure. In the VAC group, a computer-controlled pump unit was set to exert negative pressures of -75 to $-125 \mathrm{mmHg}$ equally over the entire wound bed. The VAC sponge was renewed 3 times a week, at least until the wound was free from microbiological bacterial cultures. Sternal reconstruction was planned according to the presence of repeated negative bacterial cultures, no fever, falling C-reactive protein levels (CRP $<60 \mathrm{mg} / \mathrm{L})$ and normalization of the white blood cell count.

In the BPMMF group, after the diagnosis of DSWI (Figure 1), all sternal wires as well as devascularized and necrotic cutaneous, bone and cartilage tissues were resected (Figure 2). The wounds were cleaned and rinsed with hydrogen peroxide, $10 \%$ povidone-iodine and saline water. BPMMFs were created by separating them from their chest wall insertions to the lateral border of the pectoralis major muscle. Superiorly, the dissection was stopped at the level of the clavicle. The pectoralis minor muscle, vessels, and lateral pectoral nerve were preserved. The two muscle flaps were then advanced to the midline, and the free margins were attached together (Figure 3). To avoid dead space, at least one of the flap margins was advanced into the mediastinum if the gap between the two hemisternums were wide. Moreover, the flaps were attached to the skin by suture lines without excessive tension. Furthermore, two or three chest drain tubes were placed: one under the muscle flap and the others under the subcutaneous layer. Finally, the skin was relaxedly sutured without significant tension (Figure 4). Postoperatively, patients in the BPMMF group used chest straps to fix the chest wall for 4-7 days; patients in both groups received 2-4 weeks of intravenous antibiotics according to the antimicrobial susceptibility test, followed by at least 2 weeks of oral antibiotics. The surgical procedure was shown by video in detail (see Video 1). 


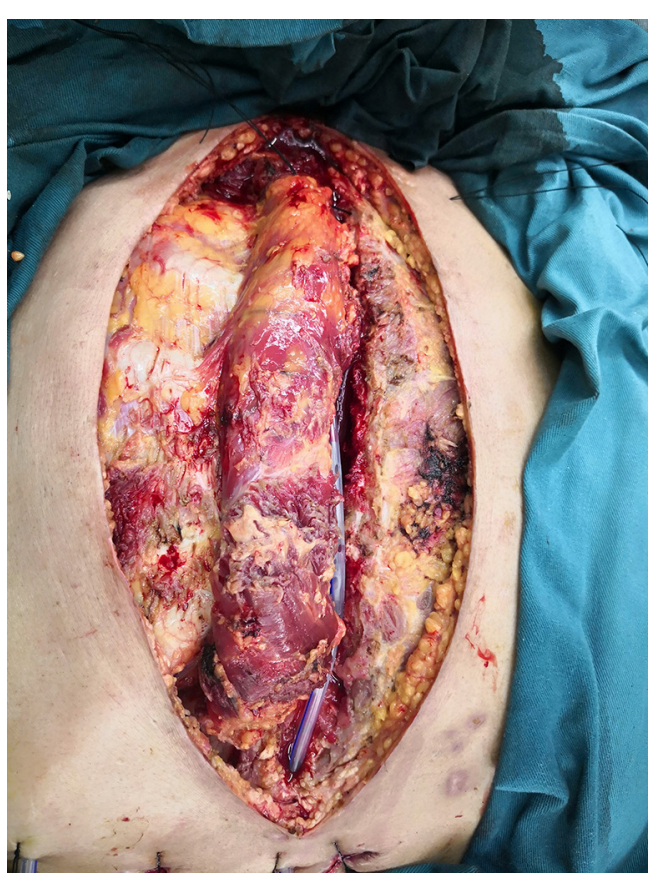

Figure 3 Bilateral pectoralis major muscle flaps: the two muscle flaps were then advanced to the midline, and the free margins were attached together.

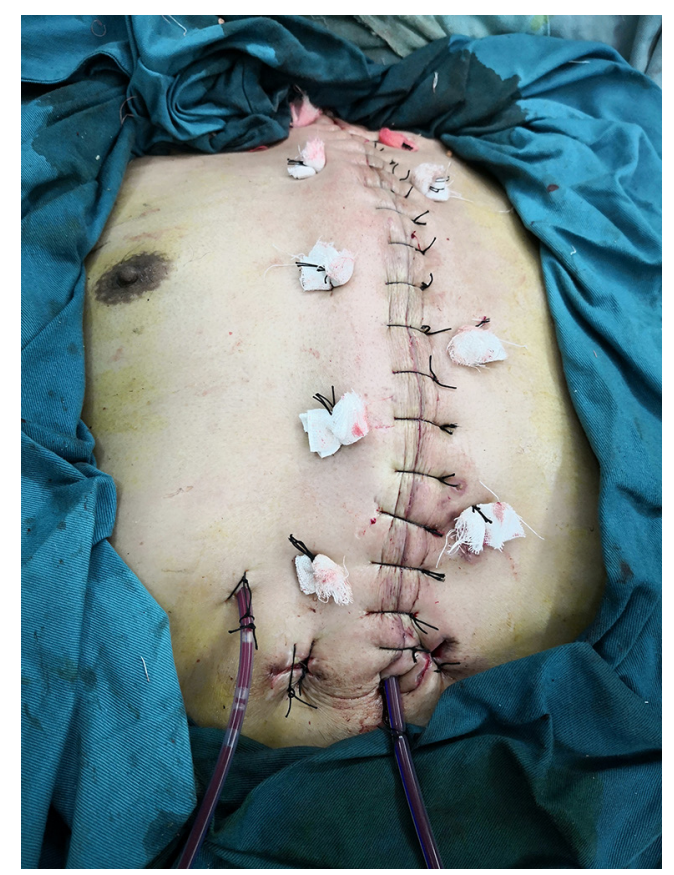

Figure 4 Bilateral pectoralis major muscle flaps: the skin was relaxedly sutured without significant tension.

\section{Data definition and collection}

The follow-up ended on October 1, 2018. In the matched cohort, patients were followed for 21.9 \pm 12.1 (median: 24, IQR: 14-28) months, and no patients were lost at follow-up. Early outcomes included length of hospital stay, uncontrolled DSWI, and death in the hospital. Late outcomes were reimplemented DSWI, death during follow-up, recurrence of DSWI and respiratory function. After repeated negative bacteriological cultures with no evidence of systemic or local infection and normalized serological inflammation parameters, patients in the VAC group underwent delayed primary closure. The BPMMF would be implemented if patients had uncontrolled DSWI or recurrence of DSWI after VAC therapy. Therefore, the definition of survival was defined as follows: patients survived, and patients in the VAC group were not converted to BPMMF placement. Therefore, patients in the VAC group who were converted to BPMMF placement were excluded from the Kaplan-Meier model. In surviving patients, variables of respiratory function included forced expiratory volume in the 1st second (FEV1), vital capacity (VC), FEV1/VC, and maximal voluntary ventilation (MVV). The data were recorded at the outpatient visit or by telephone interview.

\section{Statistical analysis}

For the statistical analysis, IBM SPSS Statistics for Windows version 22 (IBM Corporation, Armonk, NY) was used. Generally, continuous variables were described as the means \pm SDs or medians [interquartile ranges (IQRs)], as appropriate, and discrete variables were described as frequencies (n, \%). Student's t-test was used to analyze normally distributed continuous variables; nonnormally distributed continuous variables were compared using the Mann-Whitney U nonparametric method. Two groups were analyzed using repeated measures analysis of variance with various posttests whenever required. Categorical data were compared using the chi-square or Fisher's exact test, as indicated. In the matched cohort, we calculated unadjusted Kaplan-Meier survival curves showing the probability of death for each group.

We acknowledge the possibility of the existence of bias in our study. In an effort to achieve a sound scientific conclusion, we adjusted for an indication bias using a propensity score. This methodology permitted the comparison of patients who underwent BPMMF 
placement against VAC therapy with a similar risk profile. Propensity score 1-to-1 matching was utilized with the nearest neighbour algorithm without replacement and a 0.02 caliper setting (22). Age, COPD, ITAG, type of cardiac surgery, wound classification, body mass index, time before treatment for DSWI and diabetes mellitus were put into a logistic regression model to estimate the propensity score. Following matching, absolute standardized differences are used to assess pre-match imbalances and post-match balance in baseline covariates. The best balance is reflected by a standardized difference below $10 \%$. Finally, standardized mean difference was reported to assess balance (23). P values of $<0.05$ were considered to indicate a significant difference.

\section{Results}

After matching, a total of 132 patients were enrolled in this study (66 in the VAC group and 66 in the BPMMF group). In the propensity-score matched cohort, the demographic variables and wound classification were not significantly different between the two groups (Table 1). After matching, the quoted risk factors of DSWI, including age, obesity, COPD, diabetes mellitus and ITAG, were not different between the VAC group and BPMMF group (Table 1). Additionally, before propensity-score matching, the absolute standardized differences of age, COPD, ITAG, type of cardiac surgery, wound classification, body mass index, time before treatment for DSWI and diabetes mellitus were $7.1 \%, 2 \%, 1.6 \%, 10.9 \%, 166 \%, 23 \%, 80.5 \%$ and $28.7 \%$, respectively. After matching, the absolute standardized differences of these 8 variables were $3.8 \%, 7.8 \%, 4.6 \%$, $3.9 \%, 6.2 \%, 1.1 \%, 2.9 \%$ and $6.2 \%$, respectively. Therefore, these variables were well balance with a standardized difference of $<10 \%$ and had acceptable standardized mean differences (Figure 5).

The BPMMF group had the same mediastinal cultures as the VAC group (Table 2). As shown in Table 3, the BPMMF group had less septic shock $(\mathrm{P}<0.05)$ than the VAC group. Moreover, compared with VAC therapy, BPMMF placement significantly decreased the length of hospital stay $(\mathrm{P}<0.01)$. The VAC group had a higher rate of uncontrolled DSWI than the BPMMF group $(\mathrm{P}<0.05)$. The in-hospital deaths showed no significant differences between the BPMMF group and the VAC group ( $\mathrm{P}=0.4)$.

The mean duration of follow-up was $18.9 \pm 12.4$ months in the BPMMF group and $25.7 \pm 10.1$ months in the VAC group. In total, 23 patients in the VAC group received the BPMMF procedure, 11 of whom received a BPMMF because of recurrence of DSWI, and 12 of whom received a BPMMF because of uncontrolled DSWI after VAC therapy. The remaining 43 patients who had clean wounds after VAC therapy underwent delayed primary closure. Overall, the survival was $93.9 \%$ in the BPMMF group (4 patients died in the hospital, and 0 patients died during follow up) and $74.4 \%$ in the VAC group (9 patients died in the hospital, and 2 died during follow up). There were 6 patients for whom BPMMF was reimplemented in the BPMMF group due to the recurrence of DSWI. No patients in the VAC group who received a BPMMF died during follow-up. In the VAC group, the causes of hospitalized death were septic shock $(n=6)$, heart failure $(n=3)$, and the cause of late death during follow-up was multiple organ dysfunction syndrome $(n=2)$. In the BPMMF group, the causes of hospitalized death were heart failure $(n=4)$. The long-term mortality in the VAC therapy group was very high compared with that of the BPMMF group (Figure 6). The results of the postoperative respiratory function assessment are shown in Table 4. In the spirometry assessment, FEV1, VC, FEV1/ $\mathrm{VC}$ ratio and MVV showed no significant differences in survival between the VAC group and BPMMF group.

\section{Discussion}

DSWI following cardiovascular surgery is a serious and dreaded complication $(1,5)$. Its management requires repeated operations, prolonged hospitalization and increased mortality $(1,5)$. A recent report suggested that according to the available evidence, VAC is a firstline therapy for DSWI after cardiovascular surgery (11). However, some studies have reported that VAC is not able to reduce the length of hospital stay and length of ICU stay, or it is used as an adjuvant therapy $(16,17)$. Therefore, the combination of VAC and tissue flaps has been considered a justifiable treatment option for DSWI (24), but may have another demerit of high hospitalized costs and difficult manipulation. Therefore, Professor Gao developed BPMMFs to improve the treatment of DSWI. We presented a retrospective analysis of prospectively collected data that compared VAC therapy with the BPMMF procedure. This study demonstrated that (I) BPMMF was reliable and appropriate for the treatment of DSWI without any other reoperations; (II) compared with VAC therapy, BPMMF placement guaranteed better early- and late-term outcomes, as shown by less length of hospital stay and a higher rate of long-term survival; and (III) BPMMF did not 
Table 1 Demographic characteristics and wound classification according to Pairolero (20)

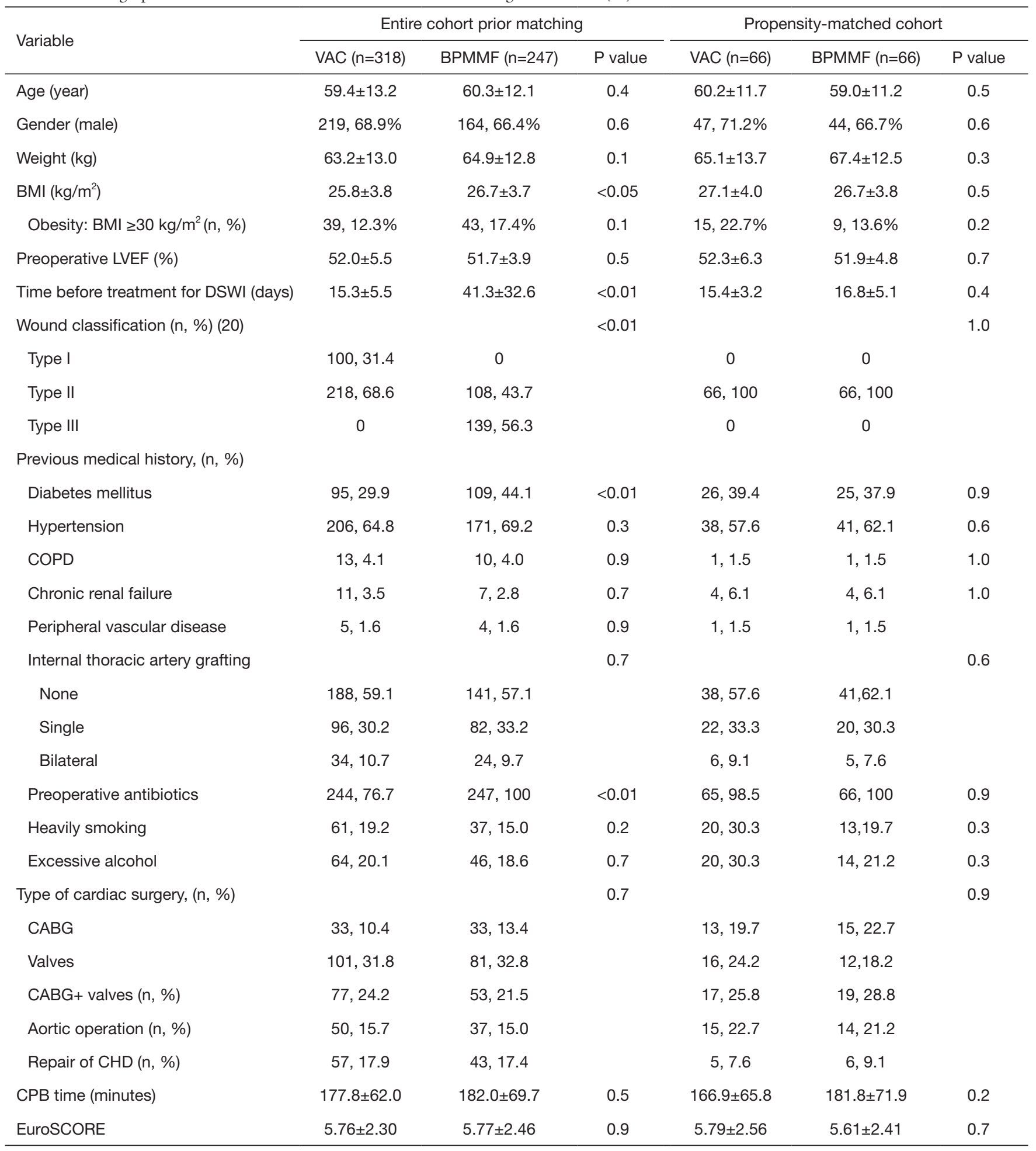

Date are presented as mean \pm SD or $(n, \%)$. BPMMF, bilateral pectoralis major muscle flap; VAC, vacuum-assisted closure; CHD, congenital heart disease; CPB, cardiopulmonary bypass; MV, mechanical ventilation; CABG, coronary artery bypass grafting; COPD, chronic obstructive pulmonary disease; LVEF, left ventricular ejection fraction. 


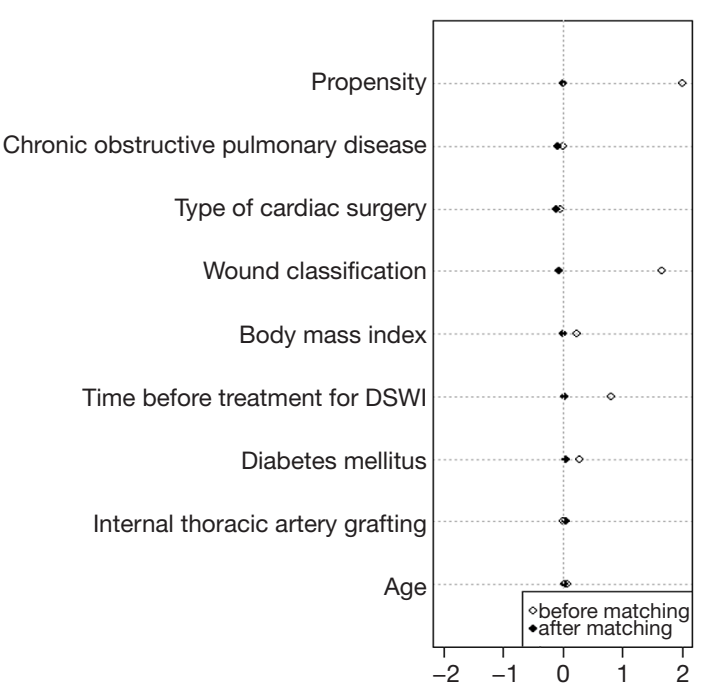

Figure 5 Covariate balance measured by standardized mean difference.

Table 2 Mediastinal cultures

\begin{tabular}{lccc}
\hline $\begin{array}{l}\text { Mediastinal cultures } \\
(\mathrm{n}, \%)\end{array}$ & $\begin{array}{c}\text { VAC group } \\
(\mathrm{n}=66)\end{array}$ & $\begin{array}{c}\text { BPMMF } \\
\text { group }(\mathrm{n}=66)\end{array}$ & P value \\
\hline Staphylococcus aureus & $42,63.6$ & $37,56.1$ & 0.4 \\
MRSA & $6,9.1$ & $10,15.2$ & 0.3 \\
Staphylococcus epidermidis & $15,22.7$ & $9,13.6$ & 0.2 \\
CONS & 0 & $3,4.5$ & - \\
Enterococcus sp. & 0 & $1,1.5$ & - \\
Klebsiella pneumonia & $8,12.1$ & $4,6.1$ & - \\
Escherichia coli & $2,3.0$ & $3,4.5$ & - \\
Enterobacter sp. & 0 & $4,6.1$ & - \\
Pseudomonas aeruginosa & 0 & $5,7.6$ & - \\
Proteus vulgaris & $3,4.5$ & 0 & - \\
Corynebacterium sp. & 0 & $1,1.5$ & - \\
Serratia marcescens & 0 & $1,1.5$ & - \\
Acinetobacter sp. & $1,1.5$ & 0 & - \\
Stenotrophomonas maltophilia & 0 & $2,3.0$ & - \\
Fungal & $1,1.5$ & 0 & - \\
\hline BPMMF, bilatera pectoralis & $090 \mathrm{musc}$ & \\
\hline
\end{tabular}

BPMMF, bilateral pectoralis major muscle flap; CONS, coagulase-negative staphylococcus; MRSA, multi-resistant staphylococcus aureus; sp., species.
Table 3 Postoperative complications and outcomes in matched cohort

\begin{tabular}{|c|c|c|c|}
\hline Variable & $\begin{array}{l}\text { VAC group } \\
\qquad(n=66)\end{array}$ & $\begin{array}{l}\text { BPMMF group } \\
\quad(n=66)\end{array}$ & $P$ value \\
\hline \multicolumn{4}{|l|}{ Local complications (n, ) } \\
\hline Skin necrosis & $1,1.5$ & $5,7.6$ & 0.1 \\
\hline Discharging sinus & $15,22.7$ & $2,3.0$ & $<0.01$ \\
\hline Partial flap loss & $1,1.5$ & $1,1.5$ & 1.0 \\
\hline Skin graft requirement & $1,1.5$ & $4,6.1$ & 0.2 \\
\hline Seroma & $11,1.7$ & $4,6.1$ & 0.06 \\
\hline \multicolumn{4}{|l|}{ Systemic complications (n, ) } \\
\hline Septic shock & $6,9.1$ & 0 & $<0.05$ \\
\hline New arrhythmia & $1,1.5$ & $8,12.1$ & $<0.05$ \\
\hline New renal failure & $6,9.1$ & $9,13.6$ & 0.4 \\
\hline \multicolumn{4}{|l|}{ Early outcomes } \\
\hline MV time (hours) & 19 [23-29] & 17 [11-22] & 0.4 \\
\hline ICU stay time (days) & $3[3-5]$ & 4 [3-4] & 0.3 \\
\hline $\begin{array}{l}\text { Length of hospital stay } \\
\text { (days) }\end{array}$ & $30[22-47]$ & 16 [14-23] & $<0.01$ \\
\hline Uncontrolled DSWI (n, ) & $12,18.2$ & 0 & $<0.05$ \\
\hline Death in hospital (n, ) & $9,13.6$ & $4,6.1$ & 0.1 \\
\hline \multicolumn{4}{|l|}{ Late outcomes (n, ) } \\
\hline Re-implemented BPMMF & $23,34.8^{*}$ & $6,9.1$ & $<0.01$ \\
\hline Death during follow up & $2,3.0$ & 0 & 0.1 \\
\hline Recurrence of DSWI & $11,1.7$ & $6,9.1$ & 0.2 \\
\hline
\end{tabular}

Date are presented as median [interquartile range] or $(n,)^{2}$ *, patients of VAC group was converted to BPMMF because of uncontrolled DSWI and/or recurrence of DSWI. DSWI, deep sternal wound infection; MV, mechanical ventilation; ICU, intensive care unit.

impair respiratory function.

There are many different therapeutic options for DSWIs in many medical centers: debridement, open packing, VAC therapy, and primary closure with irrigation followed by muscle flaps, as proposed by previous studies $(10,24,25)$. A retrospective study progressively introduced VAC therapy, followed by pectoralis myocutaneous flaps, and managed to lower the mortality rate for DSWI below 5\% (10). VAC therapy has been recommended as an important adjunct to the treatment of DSWI (17). Aggressive sternal debridement followed by VAC therapy and secondary closure with 


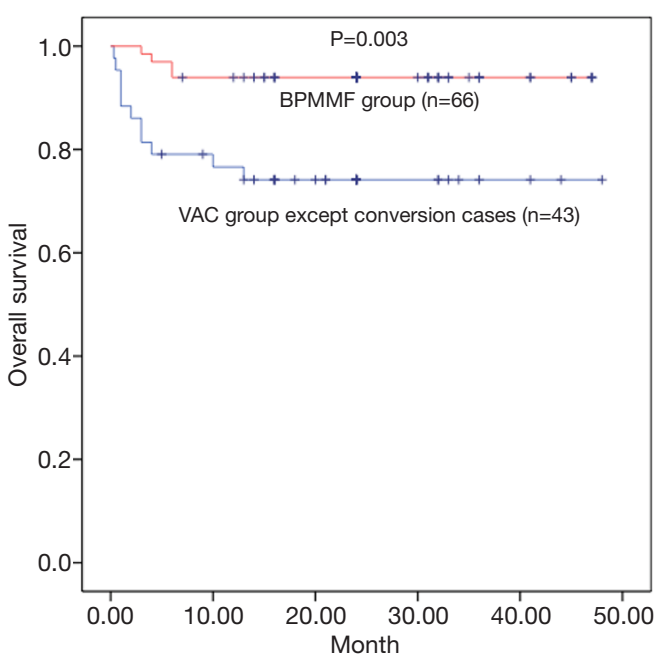

Figure 6 Kaplan-Meier survival curves at 4 years, including deaths, was $94 \%$ for the bilateral pectoralis major muscle flaps group (BPMMF; red line), and 74\% for vacuum-assisted closure therapy (VAC therapy; blue line). BPMMF, bilateral pectoralis major muscle flap; VAC, vacuum-assisted closure.

Table 4 Postoperative respiratory function data in survived patients

\begin{tabular}{lccc}
\hline Variable & VAC group $(n=32)$ & BPMMF group $(n=62)$ & $P$ value \\
\hline FEV $_{1}(\mathrm{~L})$ & $2.5 \pm 0.6$ & $2.4 \pm 0.5$ & 0.8 \\
FEV $_{1}(\%)$ & $79.2 \pm 6.6$ & $82.8 \pm 9.9$ & 0.1 \\
VC (L) & $3.3 \pm 0.5$ & $3.3 \pm 0.6$ & 0.9 \\
VC (\%) & $85.0 \pm 8.0$ & $87.4 \pm 8.8$ & 0.3 \\
FEV 1 NC (\%) & $75.4 \pm 13.6$ & $74.6 \pm 10.3$ & 0.6 \\
MVV (L/min) & $89.2 \pm 12.5$ & $85.4 \pm 18.5$ & 0.4 \\
MVV (\%) & $80.2 \pm 9.5$ & $77.4 \pm 12.6$ & 0.3 \\
\hline
\end{tabular}

Date are presented as mean \pm SD. FEV1, forced expiratory volume in the 1st second; VC, vital capacity; MVV, maximal voluntary ventilation.

an omental muscle flap is effective for DSWI (15). However, these studies do not report what stages of DSWI occurred in their study populations. As a result, there is currently a lack of consensus on the optimal strategies for different stages of DSWI. Furthermore, these previous studies suggested a "multistep" strategy that consists of massive sternal debridement, prolonged antibiotic therapy, VAC therapy and a BPMMF procedure. This approach may prolong hospitalization and create multi-resistant bacteria. Our study reported that 23 matched patients in the VAC group had to undergo BPMMF placement because of uncontrolled DSWIs and the recurrence of DSWI and that BPMMF could significantly decrease mortality. Moreover, the mean duration of hospital stay $(18.0 \pm 7.3$ days $)$ was shorter than the duration reported in an earlier study (33.7 \pm 16.5 days) (17). In other words, our "one-step" BPMMF procedure can effectively cure DSWI.

Compared with previous BPMMFs reported by earlier studies $(10,15,17)$, our BPMMFs yielded better postoperative outcomes. This may be because our BPMMF procedure has some differences. Eyileten et al. reported that their BPMMF procedure was as follows (17): (I) all infected material and sternal wires were aggressively removed; (II) an absorbable polyglycolic acid suture was used to approximate the sternal halves; (III) the medial ends of the flaps were secured at the midline with running sutures; (IV) multiple Redon catheters were placed above the pectoral muscle flaps and connected to closed drainage aspiration; (V) the skin was sutured without excessive tension. Baillot et al. (10) reported that the edge of the manubrium and the sternum were reapproximated with clamps and a minimum of three 2.4-mm-thick titanium plates in patients who underwent BPMMF placement. However, in our study, the flaps were attached to the skin as well as the sternal midline by interrupted vertical mattress suture. This method may minimize skin-muscle tension and dead space. As a result, our BPMMFs could avoid titanium plates and complex sutures, thereby benefitting the callus growth and infection control. During the BPMMF procedure, we advocate strict control of infection to protect the flaps. Therefore, aggressive debridement and aseptic procedures were initially implemented, which could provide a relatively aseptic condition to make pectoralis flaps.

Theoretically, the use of thoracic muscles for wound reconstruction might lead to impaired respiration due to greater chest rigidity. However, some previous studies have reported that surgical techniques to repair sternal wound dehiscence do not impair chest motion and respiratory function $(26,27)$. Zeitani et al. suggested that better ventilatory dynamics and a better quality of life are achieved following pectoralis muscle flap repair (26). Although the lack of preoperative respiratory assessment denied us the possibility of assessing pre- to postoperative changes, our study results indicated that the respiratory function did not differ between the BPMMF group and the VAC group. Furthermore, considering the patient population with a low incidence of COPD, this condition may not have a detrimental effect on respiratory function. 
Therefore, our study added evidence that BPMMFs allowed normal respiratory function as well as better short- and long-term outcomes. In conclusion, compared with VAC therapy, BPMMF procedures can be performed safely with satisfactory early and late results in patients with DSWIs. Meanwhile, the use of BPMMFs for sternal reconstruction not only yielded better outcomes but also allowed unimpaired respiratory function in comparison with the corresponding results of VAC therapy.

\section{Study limitations}

Our study design involved two centers' experiences with the inherent disadvantages of a retrospective study, which is highly prone to bias. This observational study could have been influenced by potential biases. We used propensity score matching to avoid these biases. However, factors that affect assignment to treatment and outcomes but that cannot be observed could not have been accounted for in the matching procedure. Any hidden bias due to latent variables might have remained after matching, which could have led to some statistical errors. Furthermore, with this analysis, we removed a large number of patients from the analysis but may have elevated the statistical errors. Finally, in the initial cohort VAC group were Type I and Type II wounds whereas pectoral flaps were type II and III. However, in the propensity score matched cohort there were then only Type II in both groups. Hence, any findings from the propensity score matched analysis cannot be assumed to also apply to wounds other than Type II. Thus, the prognostic value of BPMMFs should be assessed in future studies. These limitations of this assessment are important and should be acknowledged.

\section{Conclusions}

Compared with VAC therapy, BPMMFs guaranteed better early- and late-term outcomes, as shown by less length of hospital stay, a higher rate of long-term survival and unimpaired respiratory function.

\section{Acknowledgments}

Funding: None.

\section{Footnote}

Conflicts of Interest: The authors have no conflicts of interest to declare.

Ethical Statement: The authors are accountable for all aspects of the work in ensuring that questions related to the accuracy or integrity of any part of the work are appropriately investigated and resolved. This study was approved by ethics committee of Nanjing Drum Tower Hospital (No. 2019-244-01).

Open Access Statement: This is an Open Access article distributed in accordance with the Creative Commons Attribution-NonCommercial-NoDerivs 4.0 International License (CC BY-NC-ND 4.0), which permits the noncommercial replication and distribution of the article with the strict proviso that no changes or edits are made and the original work is properly cited (including links to both the formal publication through the relevant DOI and the license). See: https://creativecommons.org/licenses/by-nc-nd/4.0/.

\section{References}

1. Loop FD, Lytle BW, Cosgrove DM, et al. Maxwell Chamberlain memorial paper. Sternal wound complications after isolated coronary artery bypass grafting: early and late mortality, morbidity, and cost of care. Ann Thorac Surg 1990;49:179-86.

2. Kubota H, Miyata H, Motomura N, et al. Deep sternal wound infection after cardiac surgery. J Cardiothorac Surg 2013:8:132-7.

3. Ridderstolpe L, Gill H, Granfeldt H, et al. Superficial and deep sternal wound complications: incidence, risk factors and mortality. Eur J Cardiothorac Surg 2001;20:1168-75.

4. Cowan KN, Teague L, Sue SC, et al. Vacuum-assisted wound closure of deep sternal infections in high-risk patients after cardiac surgery. Ann Thorac Surg 2005, 80:2205-12.

5. Filsoufi F, Castillo JG, Rahmanian PB, et al. Epidemiology of deep sternal wound infection in cardiac surgery. $\mathrm{J}$ Cardiothorac Vasc Anesth 2009;23:488-94.

6. Borger MA, Rao V, Weisel RD, et al. Deep sternal wound infection: risk factors and outcomes. Ann Thorac Surg 1998;65:1050-6.

7. Parisian mediastinitis Study Group. Risk factors for deep sternal wound infection after sternotomy: a prospective, multicenter study. J Thorac Cardiovasc Surg 1996;111:1200-7.

8. Johnson JA, Gall WE, Gundersen AE, et al. Delayed primary closure after sternal wound infection. Ann Thorac 
Surg 1989;47:270-3.

9. Ringelman PR, Vander Kolk CA, Cameron D, et al. Longterm results of flap reconstruction in median sternotomy wound infections. Plast Reconstr Surg 1994;93:1208-14.

10. Baillot R, Cloutier D, Montalin L, et al. Impact of deep sternal wound infection management with vacuum-assisted closure therapy followed by sternal osteosynthesis: a 15year review of 23,499 sternotomies. Eur J Cardiothorac Surg 2010, 37:880-7.

11. Yu AW, Rippel RA, Smock E, et al. In patients with poststernotomy mediastinitis is vacuum-assisted closure superior to conventional therapy? Interact Cardiovasc Thorac Surg 2013;17:861-5.

12. Sjogren J, Malmsjo M, Gustafsson R, et al. Poststernotomy mediastinitis: a review of conventional surgical treatments, vacuum-assisted closure therapy and presentation of the Lund University Hospital mediastinitis algorithm. Eur J Cardiothorac Surg 2006;30:898-905.

13. Domkowski PW, Smith ML, Gonyon DL, et al. Evaluation of vacuum-assisted closure in the treatment of poststernotomy mediastinitis. J Thorac Cardiovasc Surg 2003;126:386-90.

14. Fuchs U, Zittermann A, Stuettgen B, et al. Clinical outcome of patients with deep sternal wound infection managed by vacuum-assisted closure compared to conventional therapy with open packing: a retrospective analysis. Ann Thorac Surg 2005;79:526-31.

15. Kobayashi T, Mikamo A, Kurazumi H, et al. Secondary omental and pectoralis major double flap reconstruction following aggressive sternectomy for deep sternal wound infections after cardiac surgery. J Cardiothorac Surg 2011;6:56.

16. Biefer HRC, Emmert MY, Rancic Z, et al. Negative microbiological results are not mandatory in deep sternal wound infections before wound closure. Eur J Cardiothorac Surg 2012;42:306-10.

17. Eyileten Z, Akar AR, Eryilmaz S, et al. Vacuum-assisted closure and bilateral pectoralis muscle flaps for different

Cite this article as: Pan T, Li K, Fan FD, Gao YS, Wang DJ. Vacuum-assisted closure vs. bilateral pectoralis major muscle flaps for deep sternal wounds infection. J Thorac Dis 2020;12(3):866-875. doi: 10.21037/jtd.2019.12.76 stages of mediastinitis after cardiac surgery. Surg Today 2009;39:947-54.

18. Gao YX, Wu XZ, Wang B, et al. Debridement plus bilateral pectoralis major muscle flap for treatment of sternal osteomyelitis and deep sternal wound infection. Chinese Journal of Thoracic and Cardiovascular Surgery 2001;17:113-4.

19. Liu L, Yao JM, Gao YS, et al. Curative effect of repairing transfer of pectoralis major muscle flap on chronic sternum osteomyelitis after cardiac surgery. Chinese Journal of Evidence-Based Cardiovascular Medicine 2015;6:789-91.

20. Pairolero PC, Arnold PG. Management of infected median sternotomy wounds. Ann Thorac Surg 1986;42:1-2.

21. Garner JS, Jarvis WR, Emori TG. CDC definitions for nosocomial infections 1988. Am J Infect Control 1988;16:128-40.

22. Zhang Z. Propensity score method: a non-parametric technique to reduce model dependence. Ann Transl Med 2017;5:7.

23. Zhang Z, Kim HJ, Lonjon G, et al. Balance diagnostics after propensity score matching. Ann Transl Med 2019;7:16.

24. Morisaki A, Hosono M, Murakami T, et al. Effect of negative pressure wound therapy followed by tissue flaps for deep sternal wound infection after cardiovascular surgery: propensity score matching analysis. Interact Cardiovasc Thorac Surg 2016;23:397-402.

25. Jones G, Jurkiewicz MJ, Bostwick J, et al. Management of the infected median sternotomy wound with muscle flaps. The Emory 20-year experience. Ann Surg 1997;225:766-76.

26. Zeitani J, Pompeo E, Nardi P, et al. Early and long-term results of pectoralis muscle flap reconstruction versus sternal rewiring following failed sternal closure. Eur J Cardiothorac Surg 2013;43:e144-50.

27. Kohman LJ, Auchincloss JH, Gilbert R, et al. Functional results of muscle flap closure for sternal infection. Ann Thorac Surg 1991;52:102-6. 